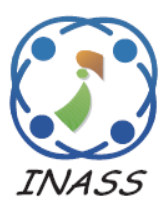

\title{
Overlay Cognitive Radio NOMA Networks with Selected Relay and Direct Link
}

\author{
Nam-Soo Kim ${ }^{1 *}$ \\ ${ }^{1}$ Deptartment of Electronic Engineering, Cheongju University, Republic of Korea \\ * Corresponding author's Email: nskim@cju.ac.kr
}

\begin{abstract}
In this paper, we propose an overlay cognitive radio (CR) non-orthogonal multiple access (NOMA) network to improve the performance of the primary receiver (PR) as well as the secondary receiver (SR). To accomplish the purpose, firstly, we adapt combining technology, selection combining (SC) and maximal ratio combining (MRC) which utilizing both signals from an indirect path and a direct path for the PR. Secondly, we select a relay which provides the spatial diversity gain among the candidate secondary sources (SSs) for the SR. The performance of the $\mathrm{PR}$ and the SR is derived in closed-form and verified through simulation. Numerical results demonstrate that the performance of the PR with SC or MRC always better than that of the PR of a conventional overlay CR NOMA system which receives from the indirect path only. Also, to maintain the same outage probability of the PR with MRC compared to that with $\mathrm{SC}$ at the given condition, $2 \mathrm{~dB}$ less transmit power is noticed. Furthermore, the performance of the SR with the selected relay improves with the increase of the number of the SSs. Consequently, we show that the proposed network, which assumes a direct link and adapts a selected relay, has better performance than the conventional overlay CR NOMA network.
\end{abstract}

Keywords: Cognitive radio, Cooperative NOMA, Direct link, Overlay CR, Selection relay.

\section{Introduction}

Recent mobile cellular systems require high throughput, reliable communication, and low latency due to rapid increase of data traffic. To satisfy these requirements, high spectral efficiency is essential [13].

In the same context, the cognitive radio (CR) has been focused on the high spectral efficiency since the licensed spectrum can be shared with a secondary system [4-7]. There are three representative CR types which are interweave, underlay, and overlay CR. Among them, underlay and overlay CR, which transmit the primary and the secondary system simultaneously with the same frequency, have higher spectral efficiency than interweave CR.

In recent studies, NOMA is applied to an overlay CR network as a secondary system because NOMA has high spectral efficiency, low latency, and mass connectivity [8-9]. Most of the overlay CR NOMA network, a NOMA relay is utilized for the performance enhancement of the Primary receiver
(PR) in shadowing areas. In [9], overlay CR with the secondary NOMA relay was considered and the information for the PR was transmitted via a NOMA relay. Also B. Chen et al., derived the performance of the PR and SR in an overlay CR NOMA network over Nakagami-m fading channels [10]. While the relay selection in overlay CR NOMA networks was investigated in [11], which have reliability-oriented and fairness-oriented schemes, it was derived the outage probabilities of PR and SR. More recently, the outage probabilities of the PR and SR in cooperative overlay CR NOMA network with channel errors and SIC errors are studied in [12]. Even though it is not a CR system, there was a study on considering the direct link for a NOMA system, but it does not include a relay selection [13].

As mentioned above, most of the studies on an overlay CR NOMA networks have been considered an indirect path only for the PR. Also for the enhanced reception probability of the PR, the more power has been allocated for the PR than a secondary receiver (SR) in NOMA processing. Moreover, further performance enhancement contributed from 
spatial diversity gain, a selected NOMA relay for the PR has been adapted. Consequently, performance improvement of the PR can be achieved by the more allocation of power and the selected relay. However, the total power of the relay is limited, hence, the more power allocation for the PR means the less power is allocated to the SR. Also, the relay selection for the PR means no spatial diversity gain to the SR. Consequently, the less power allocated to the SR and the selected relay for the PR deteriorate the performance of the SR.

Therefore, in this paper, we propose an overlay CR NOMA system to improve the performances both of the PR and SR; (1) To improve the performance of the PR, we adapt the combining technologies, Selection combining (SC) and Maximal ratio combining (MRC) which utilizing a direct path. (2) To improve the performance of the SR, we select a NOMA relay for the SR, which offers the spatial diversity gain to the SR. For the relay selection, we utilize a partial channel state information between the candidate relay-SR paths, which reduces the system overhead. The performances of the PR and the SR are derived in closed-form. The analytical outage probability expressions have been validated through simulations.

The rest of this paper is organized as follows. In section 2, the proposed overlay CR NOMA system model is presented and the transmission protocol is described as well as the outage probabilities of the PR with SC and with MRC are derived, respectively. Also the outage probability of the SR with a selected NOMA relay is derived in section 3 . The numerical examples of the outage probabilities of the PR and the SR are given, and the results are verified with the simulation in section 4. Finally, in section 5, the conclusions and the future research direction are described.

Notations: We introduce the notation used in the rest of the paper.

$h_{A B}$ : channel coefficient between node A and B, which has complex Gaussian distribution with zero mean and unit variance, $h_{A B} \sim C N(0,1)$.

$n_{A}$ : noise of node A, which has complex Gaussian distribution with zero mean and variance $N_{0}$,

$n_{A} \sim C N\left(0, N_{0}\right)$.

$\rho_{P}$ : transmit Signal-to-noise ratio (SNR) of the primary source (PS), $\rho_{P}=P_{S} / N_{0}$ where $P_{S}$ denotes transmission power of the PS.

$\rho_{R}$ : transmit Signal-to-noise ratio (SNR) of the relay, $\rho_{R}=P_{R} / N_{0}$ where $P_{R}$ denotes transmission power of the relay.
$P_{A B}$ : received power at node $\mathrm{B}$ through the node Anode B path, $P_{A B}=P_{T} d_{A B}^{-n}$ where $d_{A B}$ denotes the distance between the node $\mathrm{A}$ and the node $\mathrm{B}, P_{T}(T \in S, R)$ is the transmission power, and $n$ represents the propagation loss coefficient of the channel.

$\rho_{A B}:$ mean SNR or signal-to-noise ratio plus interference ratio (SINR), $\rho_{A B}=\rho_{A} d_{A B}^{-n}=P_{A B} / N_{0},(A \in P, R)$.

\section{System model}

Fig. 1 shows the Primary system which is composed of a primary source (PS) $P$ and a primary receiver (PR) $Q$, and the secondary NOMA system which composed of the number of $N$ secondary sources (SSs) of $S_{m}(m=1,2, \ldots, N)$ and a secondary receiver (SR) $D$. Among the SSs, a SS is selected as a relay. The selected relay is a decode-and-forward relay, which decodes the information from PS and the multiplexed signal is forwarded to the PR and to the $\mathrm{SR}$. We assume the $N$ relays are clustered, the distance and of PS-relay paths, relay-PR paths, and relay-SR paths are equal, by themselves. Each channel also has independent Rayleigh block faded, which maintain the identical channel coefficient during a time slot and independent to the next time slot.

In the first time slot, phase 1, the PS transmits and the SSs and the PR receive. The transmitted signal from the PS is $\sqrt{P_{P}} x_{P}$, where $P_{P}$ denotes the transmit

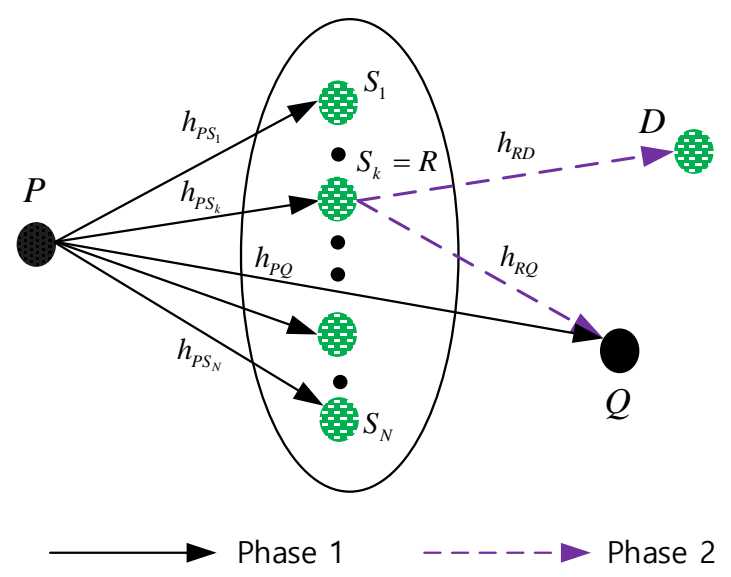

Primary system 薎 Secondary (NOMA) system

Fig.1 Proposed system model 
power of the PS, and $x_{P}$, the information to the PR, is a bipolar signal $\left|x_{P}\right|=1$ with $E\left(\left|x_{P}\right|^{2}\right)=1$. The received signal at the SSs and the PR can be written by

$$
y_{i}=\sqrt{P_{P_{i}}} h_{P_{i}} x_{P}+n_{i}
$$

where $P_{P_{i}}$ and $n_{i}\left(i \in\left\{S_{m}, Q\right\}\right)$ represent the received power through $P-i$ path and the noise of the node $i$. Denotes the subset $S$ which decodes the information from PS successfully among $N$ SSs, the cardinal of $S$ becomes $|S|=K(K \leq N)$. The best relay for the SR is selected from $S$, which has the best channel gain among $S_{I}-D(I=1,2, \ldots, K)$ paths.

The selected relay can be represented by

$$
S_{k}=\max _{I=1,2, \ldots, K}\left(\left|h_{S, D}\right|\right)
$$

Note that the selected relay can afford the spatial diversity gain for the SR, hence improves the performance of the SR, because the selection is done among the $S_{I}-D$ paths. On the other point, the selected relay is no more than a transmit node for the $\mathrm{PR}$, that does not provide the spatial diversity gain. For easy identification, we denote $R$ by the selected relay $S_{k}$.

In the second time slot, phase 2, the selected relay superimposes the information for the PR and SR using the NOMA protocol, the transmitted signal is $\sqrt{P_{R}}\left(\sqrt{\alpha_{P}} x_{P}+\sqrt{\alpha_{S}} x_{S}\right)$, where $P_{R}$ is the transmit power of the relay, and $x_{S}$, the information for the $\mathrm{SR}$, is a bipolar signal $\left|x_{S}\right|=1$ with $E\left(\left|x_{S}\right|^{2}\right)=1 . \alpha_{P}$ and $\alpha_{S}$ denote the power allocation coefficient for the PR and for the SR, respectively, where $\alpha_{P}+\alpha_{S}=1$ and $\alpha_{P}>\alpha_{S}$. The received signal at the PR from $R-Q$ path and SR from $R-D$ path can be given as

$$
y_{j}=\sqrt{P_{R_{j}}} h_{R_{j}}\left(\sqrt{\alpha_{P}} x_{P}+\sqrt{\alpha_{S}} x_{S}\right)+n_{j}, j \in\{Q, D\}
$$

\section{Outage probability}

In this section, the outage probability of the PR and SR are derived analytically. Firstly, the outage probability of the PR with SC and with MRC is derived separately. Secondly, the outage probability of the SR which has the spatial diversity gain since the selected relay is derived.

\subsection{Outage probability of Primary receiver (PR)}

When the direct path exists, the performance of a receiver can be improved by combining the signals from the direct and the indirect paths. The most representative combining technologies are $\mathrm{SC}$ and MRC. In this subsection, we derive the outage probabilities of the PR with SC and with MRC.

\subsubsection{Selection combining (SC)}

In SC, the combiner outputs the signal of the highest SNR branch. The outage probability of the PR with SC can be written by multiplying the outage probabilities of the direct path and the indirect path, which is given by

$$
P_{O, Q_{-} S C}=P_{O, Q_{-} \text {dir }} \times P_{O, Q_{-}} \text {indir }
$$

where $P_{O, Q_{-}}$dir denotes the outage probability of the direct path $(P-Q$ path). The outage happens when the received SNR at $Q$ bellows the threshold, which is

$$
P_{O, Q_{-}} \operatorname{dir}=\operatorname{Pr}\left(\gamma_{P Q}^{x_{P}}<\Gamma_{P}\right)
$$

where $\Gamma_{P}$ represents the threshold of the PR, $\Gamma_{P}=2^{2 R_{P}}-1$, and where $P_{P}$ is the required spectral efficiency of the PR. $\gamma_{P Q}^{x_{P}}$ is the received SNR at $Q$ for decoding $x_{P}$, which can be obtained from Eq. (1), and written by

$$
\gamma_{P Q}^{x_{P}}=\frac{P_{P Q}\left|h_{P Q}\right|^{2}}{N_{0}}=\rho_{P Q}\left|h_{P Q}\right|^{2}
$$

where $\quad \rho_{P Q}=P_{P Q} / N_{0}=\rho_{P} d_{P Q}^{-n} \quad . \quad$ The channel coefficient of $h_{P Q}$ in Eq. (6) is Rayleigh faded, the outage probability of the direct path, $P_{O, Q_{-}}$dir of Eq. (5) can be given by

$$
P_{O, Q_{-}} \operatorname{dir}=1-\exp \left(-\frac{\Gamma_{P}}{\rho_{P Q}}\right) .
$$

Because each channel is independent, the failure of the indirect path, $P-R-Q$ path, can be obtained by taking the complementary event that both paths (i.e., $P-R$ and $R-Q$ paths) are successful. 
Therefore, the outage probability of the indirect path is given by

$$
P_{O, Q_{-} \text {indir }}=1-\left(1-P_{O, P R}\right)\left(1-P_{O, R Q}\right)
$$

where $P_{O, P R}$ and $P_{O, R Q}$ represent the outage probabilities of the $P-R$ and $R-Q$ path, respectively. $P_{O, P R}$ can be obtained from Eq. (7) by replacing $\rho_{P Q}$ with $\rho_{P R}$ where $\rho_{P R}=\rho_{P} d_{P R}^{-n}$.

The received SINR (signal-to-interference plus noise ratio) at $Q$ from $R-Q$ path for decoding $x_{P}$, which can be obtained from Eq. (3), and written by

$$
\gamma_{R Q}^{x_{P}}=\frac{\alpha_{P}\left|h_{R Q}\right|^{2}}{\alpha_{S}\left|h_{R Q}\right|^{2}+1 / \rho_{R Q}}
$$

where $\rho_{R Q}=P_{R Q} / N_{0}=\rho_{R} d_{R Q}^{-n}$. Therefore, the outage probability of $R-Q$ path in Eq. (8) can be given by

$$
\begin{aligned}
P_{O, R Q} & =\operatorname{Pr}\left(\gamma_{R Q}^{x_{P}}<\Gamma_{P}\right)=\operatorname{Pr}\left(\left|h_{R Q}\right|^{2}<\frac{\Gamma_{P}}{\rho_{R Q}\left(\alpha_{P}-\Gamma_{P} \alpha_{S}\right)}\right) \\
& =1-\exp \left(-\frac{\Gamma_{P}}{\rho_{R Q}\left(\alpha_{P}-\Gamma_{P} \alpha_{S}\right)}\right), \Gamma_{P}<\frac{\alpha_{P}}{\alpha_{S}}
\end{aligned}
$$

where the second equality assumes Rayleigh fading. While the outage probability of $P_{O, P R}$ becomes $P_{O, P R}=1-\exp \left(-\Gamma_{P} / \rho P R\right)$ from Eq. (7), the outage probability of the indirect path can be obtained by replacing Eq. (10) into Eq. (8), as

$$
P_{O, Q_{-} \text {indir }}=1-\exp \left\{-\left(\frac{\Gamma_{P}}{\rho_{P R}}+\frac{\Gamma_{P}}{\rho_{R Q}\left(\alpha_{P}-\Gamma_{P} \alpha_{S}\right)}\right)\right\} .
$$

Consequently, the outage probability of the PR with SC in Eq. (4) can be obtained from Eq. (7) and Eq. (11).

\subsubsection{Maximal ratio combining (MRC)}

In MRC, the received signal is co-phased and multiplied by the branch SNR (i.e., complex-valued weighting is performed) $[14,15]$. After the weighting, two sums are formed. The combined SNR $\gamma_{M R C}$ is the sum of the direct path and indirect path, $\gamma_{P Q}^{x_{P}}+\gamma_{R Q}^{x_{P}}$. The outage event of the PR happens two cases: first, when $P-R$ path success, the combined SNRs at the PR below the threshold. Second, when International Journal of Intelligent Engineering and Systems, Vol.13, No.1, 2020
$P-R$ path fails and the received SNR at the PR from the direct path bellows the threshold. Hence, the outage probability of the PR can be written by

$$
\begin{aligned}
P_{O, Q_{-} M R C}= & \operatorname{Pr}\left(\gamma_{M R C}<\Gamma_{P}\right) \operatorname{Pr}\left(\gamma_{P R}^{x_{P}} \geq \Gamma_{P}\right) \\
& +\operatorname{Pr}\left(\gamma_{P Q}^{x_{P}}<\Gamma_{P}\right) \operatorname{Pr}\left(\gamma_{P R}^{x_{P}}<\Gamma_{P}\right)
\end{aligned}
$$

where $\gamma_{M R C}=\gamma_{P Q}^{x_{P}}+\gamma_{R Q}^{x_{P}}$, and $\gamma_{P R}^{x_{P}}=\rho_{P R}\left|h_{P R}\right|^{2}$ from (1). The first probability in Eq. (12) can be obtained from Eq. (6) and Eq. (9) as follows.

Theorem: The outage probability of the PR that combined the received signals both from the PS and the NOMA relay by MRC in an overlay CR system is given by

$$
\operatorname{Pr}\left(\gamma_{M R C}<\Gamma_{P}\right)=1-\exp \left(-\frac{\Gamma_{P}}{\rho_{R Q}\left(\alpha_{P}-\alpha_{S} \Gamma_{P}\right)}\right)-\Phi_{1}
$$

where

$$
\begin{aligned}
\Phi_{1}= & \frac{1}{\alpha_{S} \rho_{R Q}} \exp \left\{-\left(\frac{\Gamma_{P}}{\rho_{P Q}}-\frac{\alpha_{P}}{\alpha_{S} \rho_{P Q}}+\frac{1}{\alpha_{S} \rho_{R Q}}\right)\right\}, \\
& \times \sum_{k=0}^{\infty} \frac{(-1)^{k}}{k !\left(\alpha_{S} \rho_{R Q}\right)^{k}} \Phi_{2}
\end{aligned}
$$

and

$$
\begin{aligned}
\Phi_{2}= & \frac{(-1)^{k+2} p^{k+1}}{(k+1) !}\left\{\ln ((u))+\sum_{k=1}^{\infty} \frac{(-1)^{n} p^{n}\left(u^{n}-1\right)}{n n !}\right\} \\
& +\sum_{i=0}^{k} \frac{(-1)^{j} p^{i}}{(k+1) k \cdots(k+1-i)}\left(\frac{u^{i}}{u^{k+1}} e^{-p u}-e^{-p}\right)
\end{aligned}
$$

where $u=\left(\alpha_{P}-\alpha_{S} \Gamma_{P}\right) / \alpha_{P}$, and $p=\alpha_{P} / \alpha_{S} \rho_{P Q}$.

Proof: See Appendix.

The second probability in Eq. (12) is

$$
\operatorname{Pr}\left(\gamma_{P R}^{x_{P}} \geq \Gamma_{P}\right)=\exp \left(-\frac{\Gamma_{P}}{\rho_{P R}}\right) .
$$

And from Eq. (6), the third probability is given by

$$
\operatorname{Pr}\left(\gamma_{P Q}^{x_{P}}<\Gamma_{P}\right)=1-\exp \left(-\frac{\Gamma_{P}}{\rho P Q}\right) .
$$


Consequently, the outage probability of the PR with MRC can be obtained by replacing Eq. (13), Eq. (16), and Eq. (17) into Eq. (12).

\subsection{Outage probability of Secondary receiver (SR)}

As mentioned earlier, the power is allocated more for the message of the PR in overlay CR. This means less allocation of power for the SR since the transmit power is limited, hence the performance is degraded. This degradation can be improved by adapting the relay selection in Eq. (2) that offers the spatial diversity gain.

The outage of the SR happens two cases: First, all SSs fails to decode the information from PS successfully (i.e., $|S|=0$ ). Second, though the relay decodes the information from the PS successfully, the PR fails. Therefore, the outage probability of the SR can be written by

$$
P_{O, D}=\operatorname{Pr}(|S|=0)+\sum_{l=1}^{N} P_{O, R D}|| S \mid=I \operatorname{Pr}(|S|=I)
$$

where $\operatorname{Pr}(|S|=0)$ denotes the probability of the all SSs fails, which is given as

$$
\operatorname{Pr}(|S|=0)=\operatorname{Pr}\left(\gamma_{P S_{i}}<\Gamma_{P}\right)=\left(1-e^{-\frac{\Gamma_{P}}{\rho_{P R}}}\right)^{N}, i=1,2, \ldots, N
$$

where the second equality assumes Rayleigh fading and where $\rho_{P S_{i}}=\rho_{P R}$ which is defined in Eq. (8).

The probability of $\operatorname{Pr}(|\mathrm{S}|=l)$ in Eq. (18) denotes the probability of the number of successful decoding SSs is $l$, it becomes Bernoulli trials which is given by

$$
\operatorname{Pr}(|S|=I)=\left(\begin{array}{c}
N \\
I
\end{array}\right)\left\{\operatorname{Pr}\left(\gamma_{P R}^{x_{P}} \geq \Gamma_{P}\right)\right\}^{\prime}\left\{1-\operatorname{Pr}\left(\gamma_{P R}^{x_{P}} \geq \Gamma_{P}\right)\right\}^{N-1}
$$

where $\gamma_{P R}^{x_{P}}$ is the SNR at the relay for decoding $x_{P}$, $\gamma_{P R}^{x_{P}}=\left|h_{P R}\right|^{2} \rho_{P R}$. Then, the first probability of Eq. (20) becomes

$$
\operatorname{Pr}\left(\gamma_{P R}^{x_{P}} \geq \Gamma_{P}\right)=\operatorname{Pr}\left\{\left|h_{P R}\right|^{2} \geq \frac{\Gamma_{P}}{\rho_{P R}}\right\}=e^{-\frac{\Gamma_{P}}{\rho_{P R}}} .
$$

$$
\operatorname{Pr}(|S|=I)=\left(\begin{array}{c}
N \\
l
\end{array}\right)^{-\frac{\Gamma_{P}}{\rho_{P R}} l}\left\{1-e^{-\frac{\Gamma_{P}}{\rho_{P R}}}\right\}^{N-I} .
$$

The second probability of $P_{O, R D|| S \mid=I}$ in Eq. (18) is the outage probability of the SR which is the NOMA receiver under the given condition of $|S|=l$. The NOMA receiver cancels the interference component of the multiplexed signal using SIC prior to decode its own information. Therefore the outage happens in two cases, when the decoding the message of the PR fails and when the decoding of the information for the SR fails though the decoding of the PR success. The conditional outage probability of the SR can be given by [16]

$$
\begin{aligned}
P_{O, R D \| S \mid=l} & =\operatorname{Pr}\left(\gamma_{R D}^{x_{P}}<\Gamma_{P} \| S \mid=l\right) \\
& +\operatorname{Pr}\left(\gamma_{R D}^{x_{P}} \geq \Gamma_{P}, \gamma_{R D}^{x_{S}}<\Gamma_{S} \| S \mid=l\right)
\end{aligned}
$$

where $\Gamma_{S}=2^{R_{S}}-1$, and $R_{S}$ denotes the required spectral efficiency of the SR. Also $\gamma_{R D}^{x_{P}}$ is the SINR at the SR for decoding of $x_{P}$, it is obtained from Eq. (3),

$$
\gamma_{R D}^{x_{P}}=\frac{\alpha_{P}\left|h_{R D}\right|^{2}}{\alpha_{S}\left|h_{R D}\right|^{2}+1 / \rho_{R D}}
$$

where $\rho_{R D}=\rho_{R} d_{R D}^{-n}$. Similarly, $\gamma_{R D}^{\chi_{S}}$ is the SINR at the SR for decoding of $x_{S}$ after SIC, it is obtained from Eq. (3),

$$
\gamma_{R D}^{x_{S}}=\alpha_{S} \rho_{R D}\left|h_{R D}\right|^{2}
$$

By replacing Eqs. (24) and (25) into Eq. (23), and after rearrangement, we can write the conditional outage probability of the SR by Eq. (26) at the bottom of the next page [12]. The second equality of (26) assumes that each channel is independent and Rayleigh faded.

Consequently, the outage probability of the SR can be obtained by replacing Eq. (19), Eqs. (22) and (26) into Eq. (18).

From Eq. (21), we can obtain 


\subsection{Outage probability of the proposed system}

The outage of the proposed system in this study can be defined by the complementary event of the successful decoding both of the PR and SR, which is given by

$$
P_{O_{-} s y s}=1-\left(1-P_{O_{-} Q}\right)\left(1-P_{O_{-} D}\right)
$$

where $P_{O_{-}} Q$ denotes the outage probability of the PR, that can be replaced to $P_{O_{-}} Q_{-} S C$ in Eq. (4) with SC and $P_{O_{-}} Q_{-} M R C$ in Eq. (12) with MRC. $P_{O_{-} D}$ represents the outage probability of the SR in Eq. (18).

\section{Numerical examples}

This section describes the numerical examples of the analysis in section 3. Fig. 2 shows the outage probability of the PR with SC and MRC. In this figure, "**" denote the Monte Carlo simulation results with $1 \times 10^{8}$ iterations which show excellent match with the analytical results. For the numerical example, the distances are normalized to the distance between the PT and the PR.

It is noticed that the outage probability of the PR with SC is higher than that with MRC under the same condition. The lesser transmit power of $2 \mathrm{~dB}$ to maintain the same outage probability can be expected with MRC. For the comparison, the outage probability of the PR without direct path is denoted "Indirect path", which means the indirect path only in Eq. (11). As we expected the performance of the PR with the indirect path only is always inferior to that with the direct path irrespective of the combining technology, SC or MRC. Most of the studies on overlay CR NOMA networks have been assumed the indirect path only, however, the inclusion of the direct path signal in this study assures the performance improvement of the PR.

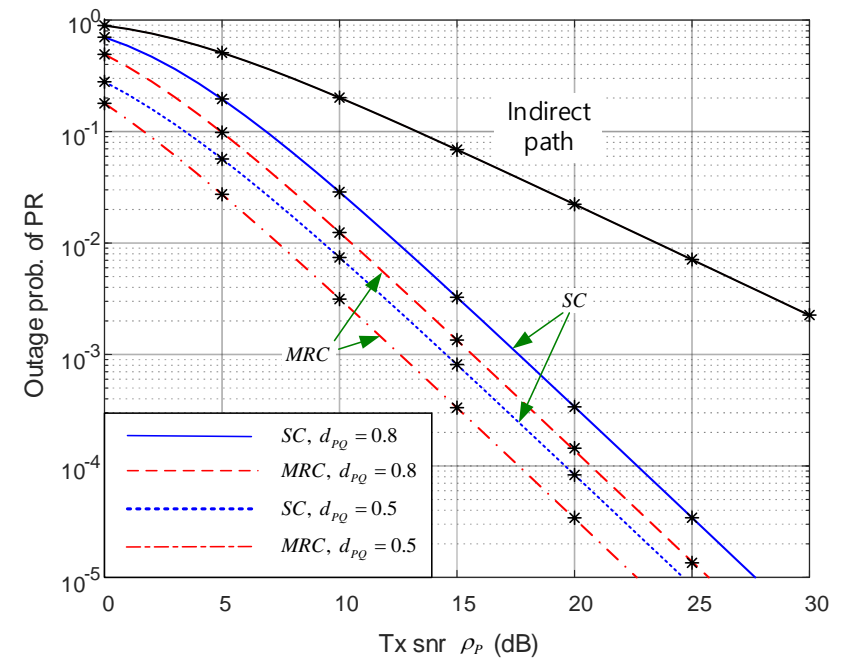

Figure. 2 Outage probability of the PR with SC or MRC $\left(n=3, d_{P R}=0.5, d_{R Q}=0.5, \alpha_{P}=0.8, \alpha_{S}=0.2, R=1\right.$, $\left.\rho_{P}=\rho_{R}\right)$

The analytical outage probabilities of the PR versus the distance between the PS and the relay are shown in Fig. 3, where "*" denote the Monte Carlo simulation results with $1 \times 10^{8}$ iterations. It is noticed that the minimum outage probability happens when the distance of the $P-R$ path is 0.7 . It is interpreted that the case with $\mathrm{SC}$, it is desirable the signal from the $R-Q$ path is stronger than that from the $P-R$ path. However, as the relay approaches to $Q$, the distance of the $R-Q$ path decreases and that of the $P-R$ path increases (i.e., $d_{R Q}=1-d_{P R}$ ). It degrades the performance of the $P-R$ path and of the indirect path, $P-R-Q$ path. Consequently, the outage probability of the PR with SC increases.

On the other hand, we noticed that the optimum distance to maintain the minimum outage probability of the PR with MRC is 0.55. Different from the performance of the PR with SC, it is interpreted that the performance with MRC is a function of the sum of the signals from the direct and indirect paths.

$$
\begin{aligned}
P_{O, R D|| S \mid=I} & =\operatorname{Pr}\left(\left|h_{R D}\right|^{2}<\max \left\{\frac{\Gamma_{P}}{\rho_{R D}\left(\alpha_{P}-\Gamma_{P} \alpha_{S}\right)}, \frac{\Gamma_{S}}{\alpha_{S} \rho_{R D}}\right\}|S|=I\right) \\
& =\left[1-\exp \left(-\max \left\{\frac{\Gamma_{P}}{\rho_{R D}\left(\alpha_{P}-\Gamma_{P} \alpha_{S}\right)}, \frac{\Gamma_{S}}{\alpha_{S} \rho_{R D}}\right\}\right)\right]^{\prime}, \quad \Gamma_{P}<\frac{\alpha_{P}}{\alpha_{R}}
\end{aligned}
$$




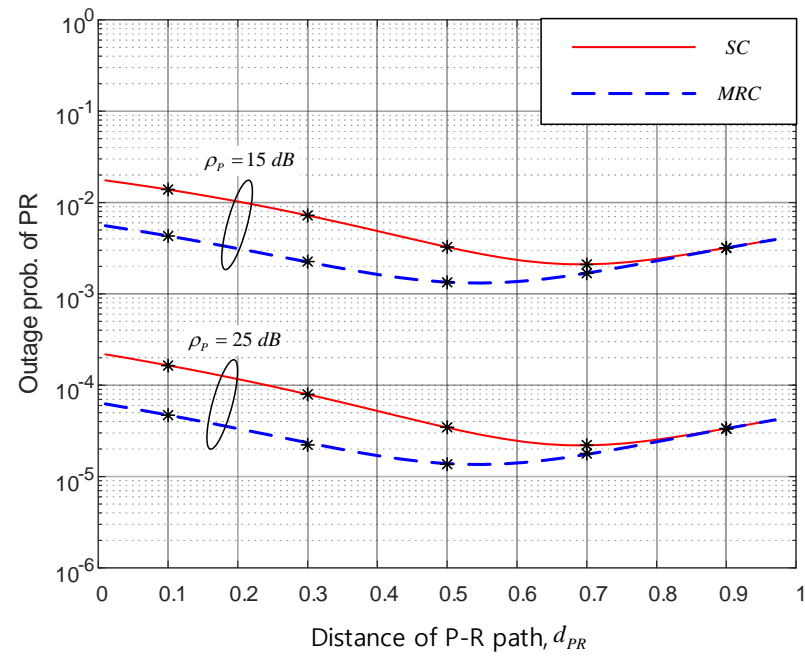

Figure. 3 Outage probability of the PR vs. distance of $P-R$ path $\left(n=3, d_{P Q}=0.8, d_{R Q}=1-d_{P Q}\right.$, $\left.\alpha_{P}=0.8, \alpha_{S}=0.2, R=1, \rho_{P}=\rho_{R}\right)$

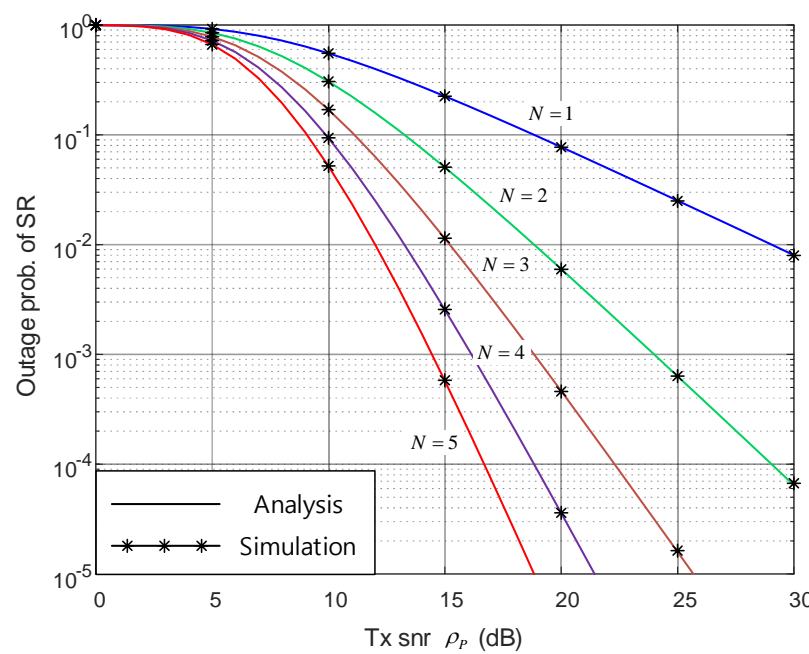

Figure. 4 Outage probability of the SR vs. number of the SSs $\left(n=3, d_{P R}=0.5, d_{R D}=0.8, \alpha_{P}=0.8, \alpha_{S}=0.2\right.$,

$$
\left.R=1, \rho_{P}=\rho_{R}\right)
$$

From this result, we conclude that the closer location of the relay to the PR than the PT is desirable, especially in the case of SC, for the better performance.

The outage probability of the SR with different number of SSs is shown in Fig. 4. Generally, the voice traffic requires an outage probability of $1 \times 10^{-3}$ but the probability without the relay selection (i.e., $N=1$ ) cannot be reached up to $30 \mathrm{~dB}$ of transmit SNR. When the number of the relays increases, the probability of $1 \times 10^{-3}$ can be obtained by the spatial diversity gain.

As the number of the SSs increases to 2, 3, 4, and 5 , the transmit SNR to satisfy the outage probability

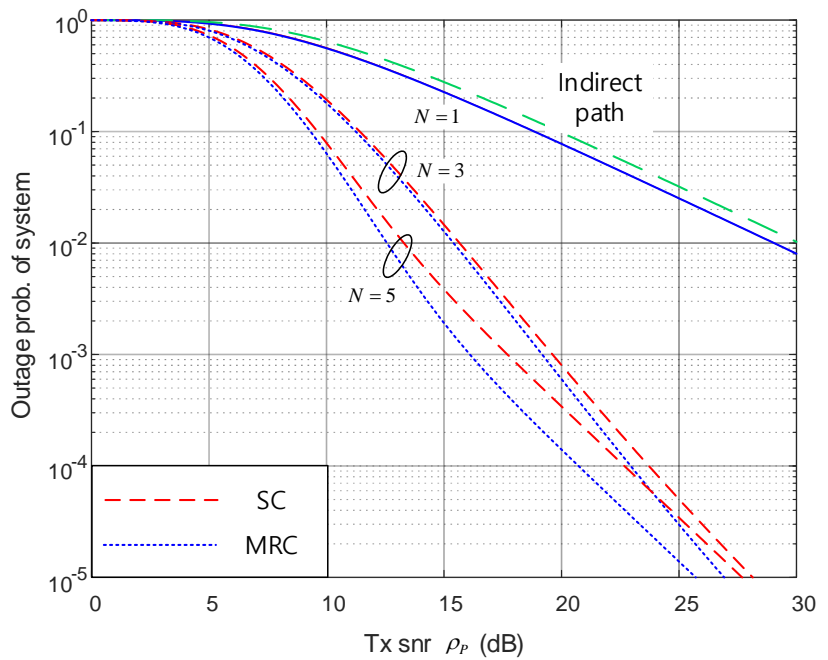

Figure. 5 Outage probability of the proposed system $\left(n=3, d_{P R}=0.5, d_{R Q}=0.5, d_{P Q}=0.8, d_{R D}=0.8\right.$, $\left.\alpha_{P}=0.8, \alpha_{S}=0.2, R=1, \rho_{P}=\rho_{R}\right)$

of $1 \times 10^{-3}$ becomes $24.0 \mathrm{~dB}, 18.9 \mathrm{~dB}, 16.1 \mathrm{~dB}$, and $14.4 \mathrm{~dB}$, respectively; the diversity gain is $15.0 \mathrm{~dB}$, $20.2 \mathrm{~dB}, 22.9 \mathrm{~dB}$, and $24.6 \mathrm{~dB}$, respectively.

The outage probability of the overlay CR NOMA system is shown in Fig. 5. When the number of the SSs is one, the outage probability of the SR is greater than the PR, hence, the outage probability of the system is close to that of the SR. However, as the number of the SSs increases, the outage probability of the system decreases; it shows that the proposed relay selection is effective for the performance enhancement. We noticed that the performance of the system with MRC is better than with SC under the same condition. The required SNR with MRC to maintain the outage probability of $1 \times 10^{-3}$ is less 0.4 $\mathrm{dB}, 1.6 \mathrm{~dB}$, and $1.9 \mathrm{~dB}$ than those with the $\mathrm{SC}$ as the number of SSs are $N=3,5$, and 7, respectively. However the SNR gain remains constant as the number of the SSs increases up to $N=8$.

In this figure, "Indirect path" curve denotes the probability of the overlay CR NOMA system with the indirect path only and no relay selection. The curve is obtained by replacing $P_{O_{-}} Q$ in Eq. (27) with $P_{O_{-}} Q_{-}$indir in Eq. (11).

This figure shows that the proposed system has better performance than the conventional "Indirect path" system.

\section{Conclusions}


In this paper, we proposed an overlay CR NOMA system with direct link and selection relay, which improves the performance of the PR and the SR. And the performance of the PR and the SR have been derived in closed-form and the analytical results were confirmed with the simulation. At the given condition, the performance of the PR with MRC is better than the PR with SC. The required SNR to maintain the same outage probability of the PR with MRC is $2 \mathrm{~dB}$ lower than the PR with SC. The results showed that the performance of the PR with either SC or MRC of the proposed system always better than the PR with the indirect path only which is the conventional assumption.

Also, we noticed that the performance of the PR was influenced by the location of the relay, when the relay is located near the PR, the performance was better than near the PS; it is noticed that the performance of the PR with SC was more sensitive to the location of the relay compared to the PR with MRC.

The numerical results showed that the performance of the SR with the selected relay improved with the increase of the number of the SSs, while the performance of the SR without the selected relay did not reach the outage probability of $1 \times 10^{-3}$ up to $30 \mathrm{~dB}$ of the transmit SNR at the given conditions. Also the performance of the proposed overlay CR NOMA system, which includes the performance of the PR and the SR, was improved.
Consequently, from those results, we can conclude that the proposed model, which includes the direct link and adapts the selected relay for the SR, is effective. The result from this study can be applied to the design of the system parameter for an overlay CR NOMA system.

Further research will be focused on the development of the new architecture of an overlay CR NOMA network for the performance enhancement.

\section{Appendix}

By replacing Eq. (6) and Eq. (9), the first probability of Eq. (12) can be written by Eq. (A.1) at the bottom of this page.

Since $\left|h_{P Q}\right|^{2} \geq 0$ in Eq. (A.1),

$\left\{\frac{\Gamma_{P}}{\rho_{P Q}}-\frac{\left|h_{R Q}\right|^{2} \alpha_{P} \rho_{R Q}}{\rho_{P Q}\left(\left|h_{R Q}\right|^{2} \alpha_{S} \rho_{R Q}+1\right)}\right\}>0$.

And after rearrangement, we can obtain

$$
\left|h_{R Q}\right|^{2}<\eta
$$

where $\eta=\Gamma_{P} / \rho_{R Q}\left(\alpha_{P}-\alpha_{S} \Gamma_{P}\right)$. Replacing Eq. (A.3) into Eq. (A.1), the probability can be written by Eq. (A.4) on the next page [17].

$$
\operatorname{Pr}\left(\gamma_{M R C}<\Gamma_{P}\right)=\operatorname{Pr}\left(\left|h_{P Q}\right|^{2}<\left\{\frac{\Gamma_{P}}{\rho_{P Q}}-\frac{\left|h_{R Q}\right|^{2} \alpha_{P} \rho_{R Q}}{\rho_{P Q}\left(\left|h_{R Q}\right|^{2} \alpha_{S} \rho_{R Q}+1\right)}\right\}\right) .
$$




$$
\begin{aligned}
\operatorname{Pr}\left(\gamma_{M R C}<\Gamma_{P}\right) & =\operatorname{Pr}\left(\left|h_{P Q}\right|^{2}<\left.\left\{\frac{\Gamma_{P}}{\rho_{P Q}}-\frac{\left|h_{R Q}\right|^{2} \alpha_{P} \rho_{R Q}}{\rho_{P Q}\left(\left|h_{R Q}\right|^{2} \alpha_{S} \rho_{R Q}+1\right)}\right\}|| h_{R Q}\right|^{2} \leq \eta\right) \\
& =\int_{0}^{\eta}\left[1-\exp \left\{-\left(\frac{\Gamma_{P}}{\rho_{P Q}}-\frac{x \alpha_{P} \rho_{R Q}}{\rho_{P Q}\left(x \alpha_{S} \rho_{R Q}+1\right)}\right)\right\}\right] e^{-x} d x \\
& =1-\mathrm{e}^{-\eta}-\int_{0}^{\eta} \exp \left\{-\left(\frac{\Gamma_{P}}{\rho_{P Q}}-\frac{x \alpha_{P} \rho_{R Q}}{\rho_{P Q}\left(x \alpha_{S} \rho_{R Q}+1\right)}+x\right)\right\} d x \\
& =1-\mathrm{e}^{-\eta}-\Phi_{1}
\end{aligned}
$$

where

$$
\Phi_{1}=\int_{0}^{\eta} \exp \left\{-\left(\frac{\Gamma_{P}}{\rho_{P Q}}-\frac{x \alpha_{P} \rho_{R Q}}{\rho_{P Q}\left(x \alpha_{S} \rho_{R Q}+1\right)}+x\right)\right\} d x .
$$

Substituting $y=x \alpha_{S} \rho_{R Q}+1$, then Eq. (A.5) becomes

$$
\begin{aligned}
\Phi_{1}= & \frac{1}{\alpha_{S} \rho_{R Q}} \exp \left\{-\left(\frac{\Gamma_{P}}{\rho_{P Q}}-\frac{\alpha_{P}}{\alpha_{S} \rho_{P Q}}+\frac{1}{\alpha_{S} \rho_{R Q}}\right)\right\} \\
& \times \int_{1}^{\eta \alpha_{S} \rho_{R Q}+1} \exp \left(-\frac{\alpha_{P}}{\alpha_{S} \rho_{P Q} y}\right) \exp \left(-\frac{y}{\alpha_{S} \rho_{R Q}}\right) d y
\end{aligned}
$$

Since $e^{x}=\sum_{k=0}^{\infty}\left(x^{k} / k !\right)$, Eq. (A.6) can be rewritten by

$$
\begin{aligned}
\Phi_{1}= & \frac{1}{\alpha_{S} \rho_{R Q}} \exp \left\{-\left(\frac{\Gamma_{P}}{\rho_{P Q}}-\frac{\alpha_{P}}{\alpha_{S} \rho_{P Q}}+\frac{1}{\alpha_{S} \rho_{R Q}}\right)\right\} \\
& \times \sum_{k=0}^{\infty} \frac{(-1)^{k}}{k !\left(\alpha_{S} \rho_{R Q}\right)^{k}} \Phi_{2}
\end{aligned}
$$

where

$$
\Phi_{2}=\int_{\left(\alpha_{P}-\alpha_{S} \Gamma_{P}\right) / \alpha_{P}}^{1} \frac{1}{z^{k+2}} e^{-\frac{\alpha_{P}}{\alpha_{S} \rho_{P Q}} z} d z
$$

From [18, 3.351.4],

$$
\begin{aligned}
\int_{u}^{\infty} \frac{e^{-p x}}{x^{n+1}} d x & =(-1)^{n+1} \frac{p^{n} E_{i}(-p u)}{n !} \\
& +\frac{e^{-p u}}{u^{n}} \sum_{k=0}^{n-1} \frac{(-1)^{k} p^{k} u^{k}}{n(n-1) \cdots(n-k)}
\end{aligned}
$$

where $E_{i}(\cdot)$ is the exponential integral, which can be substituted by $[18,8.214 .1,8.214 .2]$

$$
E_{i}(x)=C+\ln |x|+\sum_{n=1}^{\infty} \frac{x^{n}}{n n !}
$$

where $C$ is Euler's constant, $C=0.5772$. Replacing Eq. (A.9) and Eq. (A.10) into Eq. (A.8), we can rewrite

$$
\begin{aligned}
\Phi_{2}= & \int_{u}^{\infty} \frac{1}{z^{k+2}} e^{-\frac{\alpha_{P}}{\alpha_{S} \rho_{P Q}} z} d z-\int_{1}^{\infty} \frac{1}{z^{k+2}} e^{-\frac{\alpha_{P}}{\alpha_{S} \rho_{P Q}} z} d z \\
= & \frac{(-1)^{k+2} p^{k+1}}{(k+1) !}\left(\ln (|u|)+\sum_{n=1}^{\infty} \frac{(-1)^{n} p^{n}\left(u^{n}-1\right)}{n n !}\right) \\
& +\sum_{i=0}^{k} \frac{(-1)^{j} p^{i}}{(k+1) k \cdots(k+1-i)}\left(\frac{u^{i}}{u^{k+1}} e^{-p u}-e^{-p}\right)
\end{aligned}
$$

where $u=\left(\alpha_{P}-\alpha_{S} \Gamma_{P}\right) / \alpha_{P}$ and $p=\alpha_{P} / \alpha_{S} \rho_{P Q}$.

\section{References}

[1] J. G. Andrews, S. Buzzi, W. Choi, S. V. Hanly, A. Lozano, A. C. K. Soong, and J. C. Zhang, "What will 5G be?", IEEE Journal of Selected Areas in Communications, Vol. 32, No. 6, pp. 1065-1082, 2014.

[2] L. Dai, B. Wang, Y. Yuan, S. Han, C. -L. I, and Z. Wang, "Non-orthogonal multiple access for 5G: solutions, challenges, opportunities, and future research trends", IEEE Communications Magazine, Vol. 53, No. 9, pp. 74-81, 2015.

[3] C. -X. Wang, F. Haider, X. Gao, X.-H You, Y. Yang, D. Yuan, H. M. Aggoune, H. Hass, S. Fletcher, and E. Hepsaydir, "Cellular architecture and key technologies for 5G wireless communication networks", IEEE 
Communications Magazine, Vol. 52, No. 2, pp. 122-130, 2014.

[4] K. B. Letaief and W. Zhang, "Cooperative communications for cognitive radio networks", In: Proc. of the IEEE, Vol. 97, No. 5, pp. 878893, 2009.

[5] A. Goldsmith, S. A. Jafar, I. Maric, and S. Srinivasa, "Breaking spectrum gridlock with cognitive radios: an information theoretic perspective", In: Proc. of the IEEE, Vol.97, No.5, pp. 894-914, 2009.

[6] L. Lv, J. Chen, Q. Ni, Z. Ding, and H. Jiang, "Cognitive non-orthogonal multiple access with cooperative relaying: a new wireless frontier for 5G spectrum sharing", IEEE Communications Magazine, Vol. 56, No. 4, pp. 188-195, 2018.

[7] N. -S. Kim, "Performance of user relaying cooperative NOMA system underlay cognitive radio networks with partial CSI", International Journal of Intelligent Engineering and System, Vol. 12, No. 2, pp. 287-296, 2019.

[8] L. Lv, J. Chen, and Q. Ni, "Cooperative nonorthogonal multiple access in cognitive radio", IEEE Communications Letters, Vol. 20, No. 10, pp. 2059-2062, 2016.

[9] B. Chen, Yu Chen, Y. Chen, Y. Cao, N. Zhao, and Z. Ding, "A novel spectrum sharing scheme assisted by secondary NOMA relay", IEEE Wireless Communications Letters, Vo. 7, No. 5, pp. 732-735, 2018.

[10] L. Lv, Q. Ni, Z. Ding, and J. Chen, "Application of non-orthogonal multiple access in cooperative spectrum-sharing networks over Nakagami-m fading channels", IEEE Transactions on Vehicular Technology, Vol. 66, No. 6, pp. 5510-5515, 2017.

[11] L. Lv, L. Yang, H. Jiang, T. H. Luan, and J. Chen, "When NOMA meets multiuser cognitive radio: opportunistic cooperation and user scheduling", IEEE Transactions on Vehicular Technology, Vol.67, No. 7, pp. 6679-6684, 2018.

[12] N. -S. Kim, "Cooperative overlay cognitive radio NOMA network with channel errors and imperfect SIC", International Journal of Intelligent Engineering and Systems, Vol. 12, No. 5, pp. 224-231, 2019.

[13] H. Liu, Z. Ding, K. J. Kim, K. S. Kwak, and H. V. Poor, "Decode-and-forward relaying for cooperative NOMA systems with direct links", IEEE Transactions on Wireless Communications, Vol. 17, No. 12, pp. 80778093, 2018.

[14] A. Goldsmith, Wireless Communications, Cambridge University Press, New York, 2005.
[15] J. G. Proakis and M. Salehi, Digital Communications, $5^{\text {th }}$ Ed., McGraw-Hill, New York, 2008.

[16] Y. Liu, Z. Ding, M. Elkashlan, and H. V. Poor, "Cooperative non-orthogonal multiple access in 5 G systems with SWIPT", In: Proc. of the $23^{\text {rd }}$ European Signal Processing Conf. (EUSIPCO), pp. 1999-2003, 2015.

[17] X. Yue, Y. Liu, S. Kang, A. Nallanathan, and Z. Ding, "Exploiting full/half-duplex user relaying in NOMA systems", IEEE Transactions on Communications, Vol. 66, No. 2, pp. 560-575, 2018.

[18] I. S. Gradshteyn and I. M. Ryzbik, Table of integrals, series, and products, $6^{\text {th }}$ Ed., Academic Press, San Diego, CA, 2002. 